\title{
LoRaWAN Based Manhole Cover Monitoring System
}

\author{
Aman Kumar Tiwari, ${ }^{\mathrm{a}, 1}$ Priyanka Chaudhari ${ }^{\mathrm{b}}$, Shardul Pattewar ${ }^{\mathrm{b}}$, and Rohini \\ Deshmukh $^{\mathrm{b}}$ \\ a,1 UG Scholar, Dept of CSE, Sinhgad Academy of Engineering, Pune, India \\ ${ }^{b}$ Professor, Dept of CSE, Sinhgad Academy of Engineering, Pune, India
}

\begin{abstract}
An on-line monitoring system using LoRa based wireless technology for manhole cover is proposed. The system includes sensor sensing nodes, LoRaWAN network and application. LoRaWAN based IoT has very low power consumption for long-distance transmission. We use the accelerometer sensor to monitor the position, displacement or damage of manhole covers used in sewage systems. If these covers are moved or damaged, then LoRa board alerts the authorities LoRa gateway. The gateway is connected to The Things Network (TTN), a cloud-based crowd-funded open source LoRaWAN platform. The data is uploaded to the cloud and stored, and it will alert to the maintenance department. On TTN, our application will be launched and integrated with different features such as SMS.
\end{abstract}

Keywords. LoRa, LoRaWAN, Gateways, The Things Network, Manhole Cover, Smart City.

\section{Introduction}

Internet of Things and M2M communication has extremely grown in the deployment of sensor node. The development in IoT is in all stages of industry and market areas. It defines the ways of designing, managing and maintaining the networks, data, cloud and connections. IoT-enabled devices, sensors, advanced data analytics can be used for applications such as smart parking, security, agricultural farming, e-health and wearables and many more $[1,3]$. So many developments in blockchain technologies, machine learning, data analytics and artificial intelligence, there is growth in the deployments and its applications in all the sectors of industry, profession and society. Smart cities are developed so much that it made urban lives more attractive such as fast, convenient transportation systems, safe street lighting and energy-efficient buildings. IoT applications uses low complexity and energy efficient nodes for various uses on wide area networks. To support such requirements, a model of IoT, called LoRa networks was introduced. LPWAN is a wireless IoT technology which covers larger

\footnotetext{
${ }^{1}$ Priyanka Chaudhari, Department of Computer Engineering, Sinhgad Academy of Engineering, India; E-mail: c.piyu@outlook.com
} 
areas, low data rate and long battery life [3]. Low power consumption and longdistance transmission characteristics have good development anticipation in the field of IoT. In our paper, we proposed to design and develop LoRaWAN based system to monitor manhole covers used in sewage systems. In developing countries, monitoring the health of manhole cover is a challenging problem [4]. Often, manhole covers are damaged, as shown in Fig. 1 or stolen because of that several accidents and deaths. Our system will sense where about of manhole cover and its health. In case of damage or theft, it will intimate the cloud-based server in real-time, and the server will alert the concerned officers or personnel about the same so that they can take the necessary action as soon as possible.

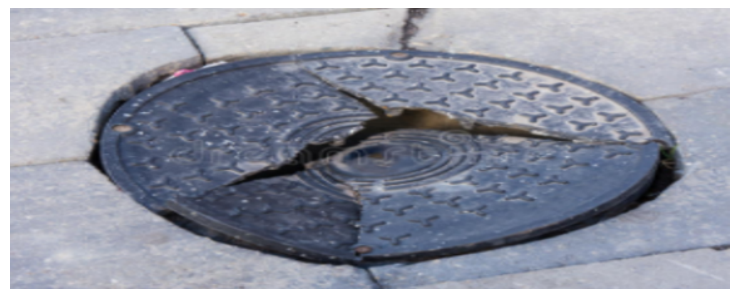

Figure 1. A damaged manhole cover

\section{LoRa WAN Technology and The Things Network}

LoRa is low power network technology. This is one of the long range and latest technologies contingent on chirp spread spectrum technology $[5,6]$. LoRa is the protocol that was developed to define the upper layers of the network. LoRa is a cloudbased MAC layer protocol, manages the communication between LPWANgateways and end node devices. The LoRa Alliance sustains LoRaWAN. The block diagram of the typical LoRaWAN network is as shown in Fig. 2.

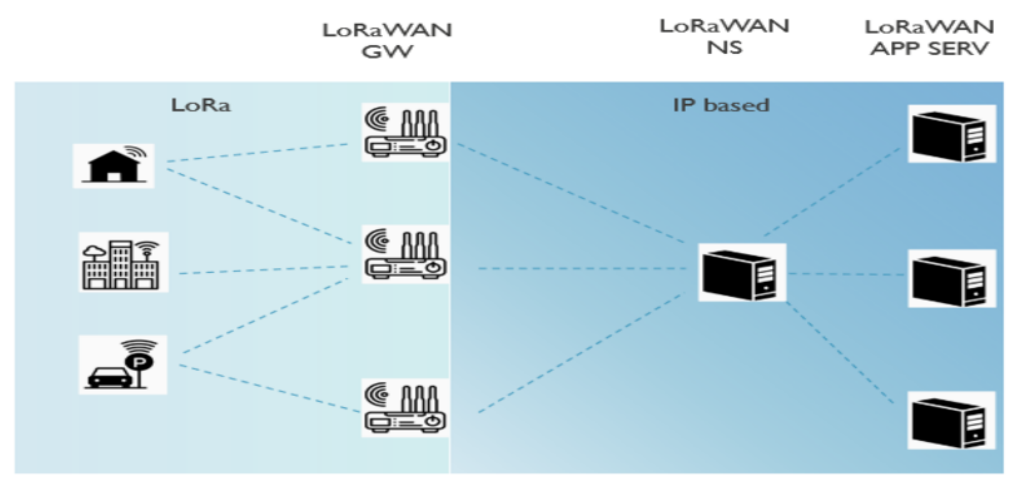

Figure 2. Block diagram of LoRaWAN network 
The Things Network is cloud-based decentralized and open-source LoRaWAN platform to exchange data with applications [7]. It connects low power end devices to gateways which may be placed far away. A TTN application retrieves many parameters such as:

- the registered devices such as sensor nodes, their health and the data they are sending in real-time

- It allows identifying nodes through application ID on the network

- general settings of the application such as network keys, security, activation type

- a payload decoder which gives instructions used for converting the incoming messages

Once application is functional, one can inscribe a device to use it. This gives the necessary IDs and security keys to connect a sensor node to the network. Once the sensor node has joined the network, we can retrieve the data it sends in real-time, either directly through other data collection services or TTN interface.

\section{Related work}

Researches and projects have been undertaken on LoRaWAN for different applications used in smart cities and other places. Green Cityzen and the United Nations secure water delivery in African refugee camps using the LoRaWAN project aims to secure 15 camps hosting nearly 2 million refugees in Uganda and Ethiopia. The GreenCityzen Business application manages the fleet of sensors, monitors the key indicators, generates alerts, and helps make the best decisions and control invoicing of their water tank suppliers. The TTN deploys a LoRaWAN network, while the French start-up has installed a network of 300 Ultrasonic Level sensors with an IoT platform that continuously monitorsavailable water stocks and truck deliveries [5]. Smart electric meter using LoRa aims to take electric energy consumption readings reading periodically and send it to the Internet for monitoring and control [7]. A smart prepaid electric meter with LoRa module measures the data such as energy power, voltage etc. and sends it to a gateway. Gateway is the central unit which collects all the data from all the meters, and it is connected with the local storage device. The gateway sends the data to the cloud [9].

\section{Implementation}

In this paper, we propose to design and develop LoRaWAN based system to monitor manhole covers used in sewage systems. In developing countries, monitoring the health of manhole cover is a challenging problem. Many times, manhole covers are damaged or stolen because of that several accidents and deaths. Our system will sense where about of manhole cover and its health. In case of damage or theft, it will intimate the cloud-based server in real-time, and the server will alert the concerned officers or personnel about the same so that they can take the necessary action as soon as possible.

We are using LoRa nodes, accelerometer sensors, LoRa gateway, and TTN server. LoRa nodes and gateway are programmed and configured by using python. In the manhole cover monitoring system, the accelerometer sensors sense the damage and 
theft of cover using angle algorithm periodically. When there is the movement of manhole cover, it alerts the LoRa board connected there. LoRa board transmit RF signal wirelessly in the unlicensed frequency band for long-distance. This signal is received by nearby LoRa nano-gateway which is connected to TTNserver. The data will be uploaded to the cloud and stored, and it will alert the maintenance department for repairing purpose. The block diagram of the system is as shown in Fig. 3.

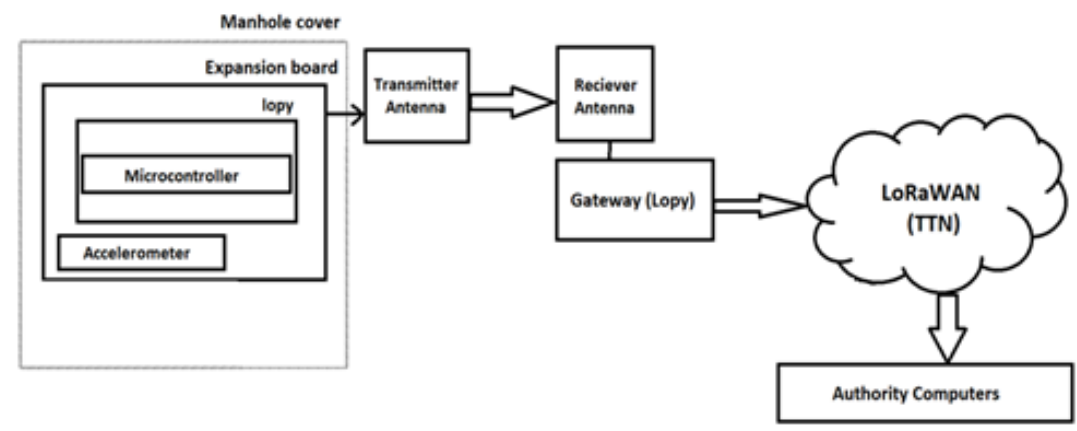

Figure 3. Block schematic of manhole cover monitoring system

During the implementation, we have used LoRa nodes from PyCom, which have inbuilt the accelerometer sensor [8]. The nodes are programmed by using micro Python and Pymakr. PyCom nodes can also be configured as single-channel nano gateways for LoRaWAN. We have programmed one of the nodes as LoRa nano gateway to receive the LoRa signal from sensing LoRa nodes and hence the health of manhole covers. The Nano gateway is connected to the Internet so that it can send data to the TTN server. On TTN server, we have configured an application to decode the data and integrated a service to send email and SMS. When the manhole cover is abnormal, it will send out an alarm message to notify the relevant departments to repair and realize the municipal manhole cover's periodical supervision. The proposed placement of the LoRa node on the manhole cover is as shown in Fig. 4. The process flowchart for the working is, as shown in Fig. 5.

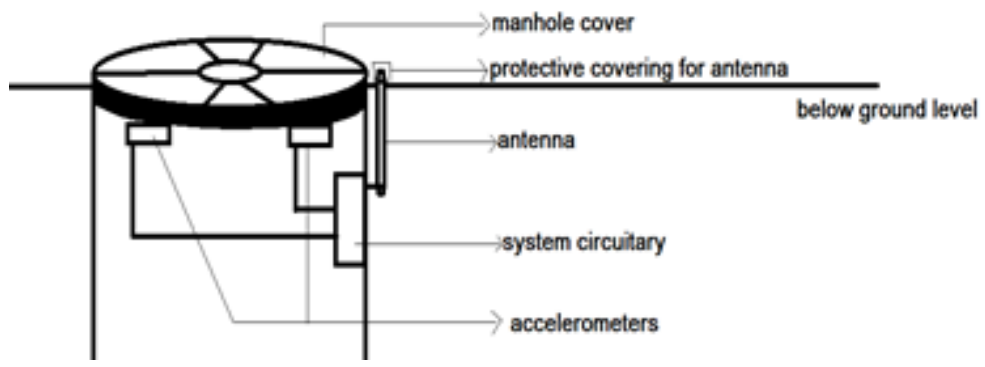

Figure 4. Placement of LoRa node on manhole cover 


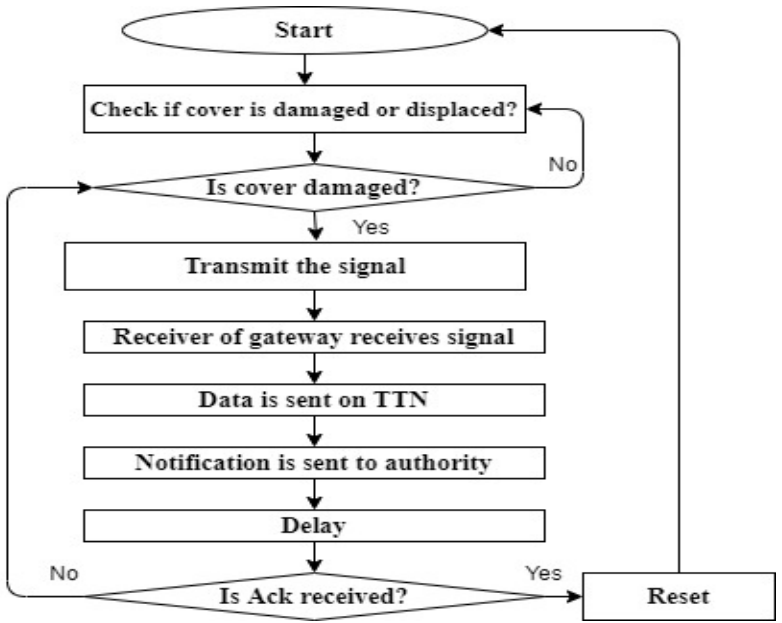

Figure 5. Process flowchart for manhole cover monitoring system

\section{Conclusion}

We propose the use of low-power long-distance LoRaWANtechnology for manhole cover monitoring. This low cost and highly secured technology have the advantage of long-distance transmission with unlicensed band operation. In case of any damage, displacement or theft of manhole cover, the system immediately alerts the maintenance department for the corrective action. The system can avoid accidents as well as saves the lives of the citizens. This technology can be used for many smart cities and other applications for the betterment of society.

\section{References}

[1] Chaudhari, Bharat S. and Marco Zennaro, eds. LPWAN Technologies for IoT and M2M Applications. Academic Press, 2020.

[2] Adelantado, Ferran, Xavier Vilajosana, Pere Tuset-Peiro, Borja Martinez, Joan Melia-Segui, and Thomas Watteyne. Understanding the limits of LoRaWAN, IEEE Communications magazine 55, no. 9, pp. 34-40, 2017

[3] Chaudhari, Bharat S., Marco Zennaro, and Suresh Borkar. LPWAN technologies: Emerging application characteristics, requirements, and design considerations. Future Internet vol. 12, no. 3, pp. 46-71, 2020.

[4] Zhang, He-sheng, Lei Li, and Xuan Liu, Development and test of manhole cover monitoring device using LoRa and accelerometer, IEEE Transactions on Instrumentation and Measurement. vol. 69, no. 5, pp. 2570-2580, 2020.

[5] Bankov, Dmitry, Evgeny Khorov, and Andrey Lyakhov, On the limits of LoRaWAN channel access. In 2016 International Conference on Engineering and Telecom (EnT), pp. 10-14. IEEE, 2016.

[6] https://www.semtech.com/lora

[7] https://www.thethingsnetwork.org/docs/index.html

[8] www.pycom.io

[9] Ambeth Kumar.V.D .Precautionary measures for accidents due to mobile phone using IOT. Clinical eHealth, Volume 1, Issue 1, March 2018, Pages 30-35.

[10] K. Sabarinathan et.al ., " Machine Maintenance Using Augmented Reality”, 3rd International Conference on Communication and Electronics Systems (ICCES), 2018. (DOI: 10.1109/CESYS.2018.8723900) 